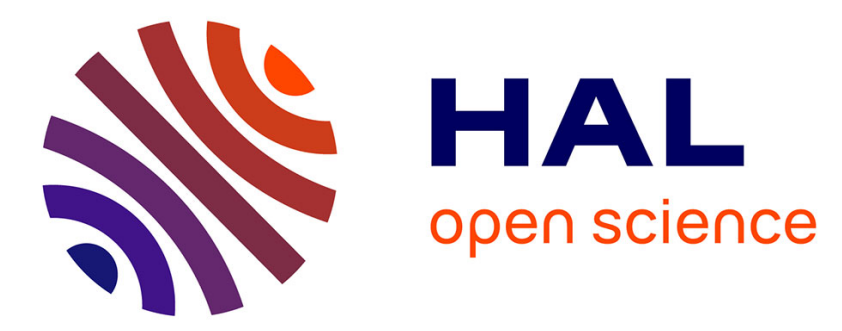

\title{
Applications of Transient Signal Analysis Using the Concept of Recurrence Plot Analysis
}

Angela Digulescu, Irina Murgan, Cornel Ioana, Ion Candel, Alexandru Serbanescu

\section{- To cite this version:}

Angela Digulescu, Irina Murgan, Cornel Ioana, Ion Candel, Alexandru Serbanescu. Applications of Transient Signal Analysis Using the Concept of Recurrence Plot Analysis. Recurrence Plots and Their Quantifications: Expanding Horizons, 180, pp.19-38, 2016, 978-3-319-29921-1. 10.1007/978-3319-29922-8_2. hal-01447912

\section{HAL Id: hal-01447912 https://hal.science/hal-01447912}

Submitted on 27 Jan 2017

HAL is a multi-disciplinary open access archive for the deposit and dissemination of scientific research documents, whether they are published or not. The documents may come from teaching and research institutions in France or abroad, or from public or private research centers.
L'archive ouverte pluridisciplinaire HAL, est destinée au dépôt et à la diffusion de documents scientifiques de niveau recherche, publiés ou non, émanant des établissements d'enseignement et de recherche français ou étrangers, des laboratoires publics ou privés. 


\title{
Applications of Transient Signal Analysis using the concept of recurrence plot analysis
}

\author{
Angela Digulescu ${ }^{1,2, *}$, Irina Murgan ${ }^{1}$, Cornel Ioana ${ }^{1, *}$, Ion Candel $^{1}$ and \\ Alexandru Serbanescu ${ }^{2}$ \\ ${ }^{1}$ Grenoble Institute of Technology, GIPSA-lab, France \\ ${ }^{2}$ Military Technical Academy, Bucharest, Romania \\ E-mail*:\{Angela.Digulescu; Cornel.Ioana\}@gipsa-lab.grenoble-inp.fr, adigulescu@mta.ro
}

\begin{abstract}
Transient signals are universally characterized by a short duration and a broad spectrum which are often present in various phenomena such as sudden acoustic pressure changes, seismic waves, electrical discharges, etc. In order to efficiently monitor the systems where they happen, it is very important that the signals generated by transient phenomena be detected, located and characterized. This significantly helps to better understand their effects in the given application context. This paper presents new tools derived from the concept of Recurrence Plot Analysis (RPA) and applied on three real applications. Two of the applications concern the detection, localization and characterization of the electrical partial discharges (measured from photovoltaic panels and on electrical cables, respectively). Another application refers to the quantification of the water hammer effect using two acoustic sensors placed on a pipe line.
\end{abstract}

\section{Introduction}

Complex systems are often met in real life and they usually present highly nonlinear (and sometimes linear) deterministic, stochastic and random characteristics [1]. These systems comprise different subparts which are strongly interconnected, hereby the interdependence of their characteristics is difficult to depict, and, therefore, the system has to be characterized as a whole and not individually. This is the reason why for most real applications, the measurements are very well suited to reveal the resultant effect of the processes that describe a phenomenon under study [2].

Hereby, the choice of the RPA concept for the analysis of transient signals is based on the fact that it is a data-driven method which does not require a priori in- 
formation about the system, knowing that such information is not always available [3].

Our applications under study concern the transient signals that appear in hydraulic and power systems. The major issue is that these signals reflect a sudden change of the dynamical system which can cause, in an unpredictable laps of time, a breakdown of the system.

The recurrence information is very important, offering us new insights in the analysis of transient signals which represent totally different states of the systems. In our work, we are interested of the system state changes that are not determined by random causes, but they are the results of a nonlinear input that causes them to change their state suddenly, exposing the system to major collapse.

The first application relates to the electrical partial discharge (PD) detection and characterization [4]. The PDs indicate that some changes have occurred in the insulation due to chemical and/or mechanical transformations [5], which, in time, can lead to the failure of the equipment. Hereby, the PD measurement is a routine procedure for testing important components from the power system (high-voltage cables, transformers, etc.).

The second application concerns the detection, localization and characterization of electrical arcs generated in photovoltaic panels [6, 7]. The need of detection, localization and characterization of the electrical arcs is a growing demand as these systems continue to develop and the environmental conditions still unexpectedly change.

Next, our application refers to the water hammer effect which appears in pipelines when a valve is suddenly closed, so it forces the fluid to change its direction or to stop its flow. This translates to a pipe pressure sudden increase/ decrease which causes from vibrations of the pipe to system collapse. Thus, this phenomenon must be supervised and characterized in order to control its damaging effects to the hydraulic system.

Through these specific applications, our paper shows the interest of RPA approaches for the analysis of the transient signals in various applications of nowadays interest.

The paper is organized as follows: the second section presents some relative new signal analysis tools based on the RPA concept. Next, each section presents the applications mentioned above and discusses on the subject. The last section illustrates the conclusions and perspectives of our work.

\section{Signal Analysis tools based on the RPA concept}

In this section, starting from the concept of recurrence plot analysis, two new analysis tools derived from RPA concept are presented.

Firstly, there will be highlighted the measure used for the detection of a transient signal, namely the time-distributed recurrence (TDR) measure. Then, the 
multi-lag phase-space analysis will be introduced. This concept is very useful for the characterization of transient signals.

\subsection{The time-distributed recurrence measure}

The basis of this measure starts from the idea that a sudden change in a time series represents a new state of the dynamical system $[3,8,9,10,11,12,13,14]$, namely there is no recurrence with the previous states. Therefore, when an appropriate distance is used, the recurrence matrix presents a horizontal/vertical band with much fewer recurrences. When the sum of the lines/ columns of the recurrence matrix is computed, we actually obtain the column average [3], which, in the case of transient signals, significantly changes.

Considering a measured signal as the following time series $[15,16,17,18,19$, 20,21]:

$$
\mathrm{s}[n]=\{s[1], s[2], \ldots, s[N]\}
$$

where $N$ is the length of the signal, then the phase-space points of the system are obtained from the available time series:

$$
\overrightarrow{v_{i}}=\sum_{k=1}^{m} s[i+(k-1) d] \cdot \overrightarrow{e_{k}}
$$

where $m$ is the embedding dimension of the phase-space, $d$ is the delay (lag) chosen between the samples of the time series and $\overrightarrow{e_{k}}$ are the axis unit vectors corresponding to each dimension of the phase-space.

Then, the distance/ recurrence matrix is obtained:

$$
R_{i, j}=\Theta\left(\varepsilon-\left\|\overrightarrow{v_{i}}-\overrightarrow{v_{j}}\right\|\right), i, j=\{1,2, \ldots, M\}
$$

where $\|\cdot\|$ is a certain chosen distance (Euclidean distance [8, 21], angular distance [3, 8], L1 norm [22], etc.) and the $\Theta(\cdot)$ is the Heaviside step function. For our applications, the threshold $\varepsilon$ is considered constant and $M=N-(m-1) d$.

Hereby, the time-distributed recurrence (TDR) measure is defines as $[3,8]$ :

$$
\operatorname{TDR}[n]=\frac{1}{M} \sum_{i=1}^{M} R_{i, n}
$$

This measure can be interpreted as the column average recurrence of a given point $i$ or the recurrence density heterogeneity in the point $i$. Hereby, a solitary position of a phase space vector changes significantly its average recurrence and can be highlighted through the use of the complementary version of the measure from eq. (4): 


$$
T D R^{*}[n]=1-\frac{T D R[n]}{\max \{T D R[n]\}}
$$

Moreover, in order to detect only the transient signal from the analyzed observation, the signal-to-noise ratio (SNR) must be computed.

The SNR is computed as follows: the last part of the acquired signal (when no phenomena is happening) is considered as noise, $z[i]$, (unwanted signal recording environmental noise, cable noise, noise caused by imperfect connections, etc.), whereas the part that has a different behavior is considered as the interest signal, $s[i]$. Both parts of the signal, $s[i]$ and $z[i]$ have the same length, $N$. The SNR is computed as:

$$
S N R=10 \log _{10}\left(\frac{\sum_{i=1}^{N}(s[i])^{2}}{\sum_{i=1}^{N}(z[i])^{2}}\right)
$$

Accordingly, the threshold $\varepsilon$ of the recurrence matrix is chosen so that it includes the a percentage, $\alpha$, of the maximum value of the equivalent noise which has the same power as the interest signal:

$$
\varepsilon=\alpha \cdot \max \left(\mid S N R_{\text {signal }} \cdot \text { noise } \mid\right)
$$

where $\alpha$ is a constant that is chosen to nonlinearly filter the noise. In our applications, $\alpha$ varies from 0.4 to 0.95 .

Considering the eq. (7), the components of the noise (undesired parts of the signal) are considered as recurrences, therefore the transient signal (useful part of the signal) is highlighted by the proposed measure.

(a)

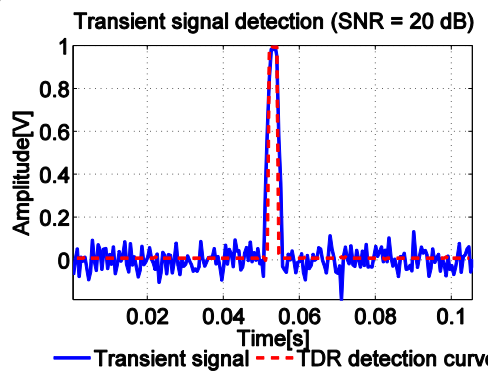

(c) (b)

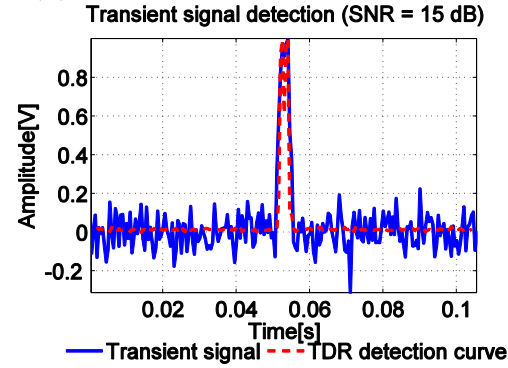

(d) 

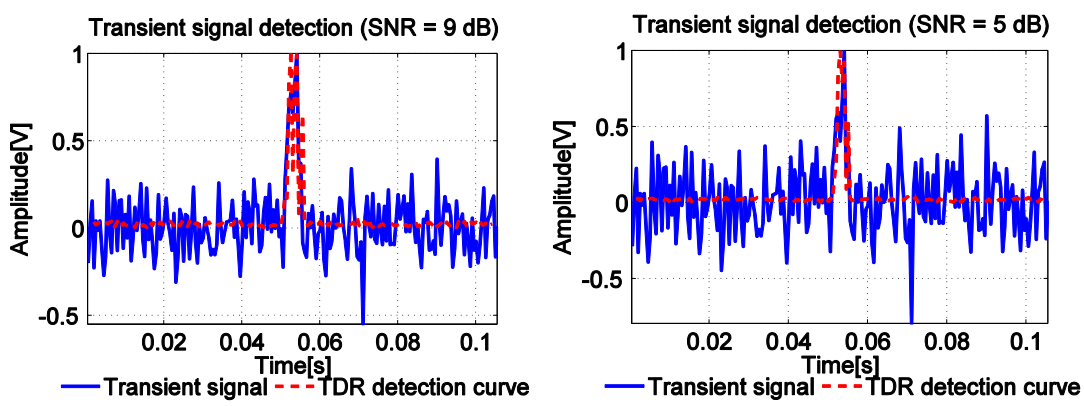

Fig. 1 Transient signal detection using the TDR measure for a transition of only 11 samples where $m=3, d=2$ and $\alpha=0.8$

Fig. 1 emphasizes the advantage brought by the TDR measure, namely that the detection provided by the detection curve is more robust to noise the actual recorded signal (electrical arc acquisition). In the examples above, the detection curve exhibits a SNR improvement of $30 \mathrm{~dB}$.

The major advantage of this method, is that, in applications where the SNR level of the acquired signal varies a lot, the TDR detection curve approach improves the SNR level after filtering the acquired signal with the detection curve. Through this approach, the signal's power after filtering is significantly increased (in our applications, at least $10 \mathrm{~dB}$ ), which is very helpful for the part of signal classification (section 3 and 4). Moreover, this provides the robustness to the TDOA (time difference of arrival) estimation. This comes from the measure's invariance to the group velocity effect (thanks to the concept of recurrence), whereas the peak detection or the correlation function is very sensitive to this effect (section 5).

\subsection{Multi-lag phase-space analysis}

The RPA method stands, as its name suggests it, on the concept of recurrences. Still, for our applications, a step backward has been made and a closer attention is given to the phase-space in order to achieve richer characterization of similar signals coming from the same source and having the same propagation and acquisition conditions.

The phase-space representation is very rich in information regarding the evolution of a dynamical system $[22,23]$, therefore the analysis can be made in any dimension, but our approach restrains, for the moment, to a bi-dimensional representation.

Therefore, considering the Cartesian coordinate system, the phase-space representation is given by: 


$$
\left\{\begin{array}{c}
x_{n}=s[n] \\
y_{n}=s[n+d]
\end{array}\right.
$$

where $n$ is the $n^{\text {th }} \quad$ sample of the recorded time series. It goes that eq. (2) can be rewritten as: $\overrightarrow{v_{n}}=s[n] \cdot \vec{i}+s[n+d] \cdot \vec{j}$.

Firstly, the main attributes of this representation are recalled. Considering three signals $s_{1}, s_{2}$ and $s_{3}$ defined as:

$$
\begin{aligned}
& s_{1}[n]=s[n+\delta] \\
& s_{2}[n]=s[\alpha n] \\
& s_{3}[n]=\beta \cdot s[n]
\end{aligned}
$$

where $\alpha, \beta$ and $\delta$ are constant which modify the signal $s$ through translation in time, scale and or amplitude, the phase-space points present the following attributes $[20,24,25]$ :

$$
\begin{aligned}
& \overrightarrow{v_{1[i]}}=\overrightarrow{v_{[i+\delta]}} \\
& \overrightarrow{v_{2[i]}}=\overrightarrow{v_{[\alpha i]}} \\
& \overrightarrow{v_{3[i]}}=\beta \cdot \overrightarrow{v_{[i]}}
\end{aligned}
$$

The phase-space trajectory is invariant to translation and it points out the scale and amplitude change.

In order to introduce the concept of multi-lag phase-space analysis, three transient signals with similar characteristics are considered. These signals are given by the generic signal:

$$
s_{(a, f, b)}[n]=\left\{\begin{array}{c}
a \cdot(\sin [2 \pi \cdot f \cdot n]+b[n]), n=\{1,2, \ldots, N\} \\
a \cdot b[n], \text { otherwise }
\end{array}\right.
$$

where $N=\left[\frac{f_{s}}{2 f}\right]$ and $f_{s}=10 \mathrm{MHz}$ is the sampling frequency.

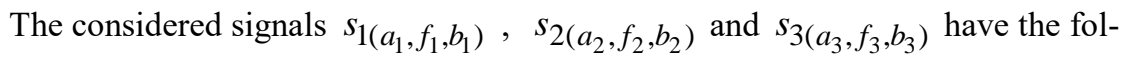
lowing relationships between their parameters: $a_{1} / a_{2}=1 / 0.6=1.66$, $a_{1} / a_{3}=1 / 0.3=3.33, \quad f_{1} / f_{2}=\left(2 \cdot 10^{5}\right) /\left(1.9 \cdot 10^{5}\right)=1.05$ $f_{1} / f_{3}=\left(2 \cdot 10^{5}\right) /\left(1.6 \cdot 10^{5}\right)=1.25, \quad b_{1}=b_{2}=b_{3} \quad$ and $\quad S N R_{i}=20 \mathrm{~dB}$, $i=\{1,2,3\}$.

(a) 

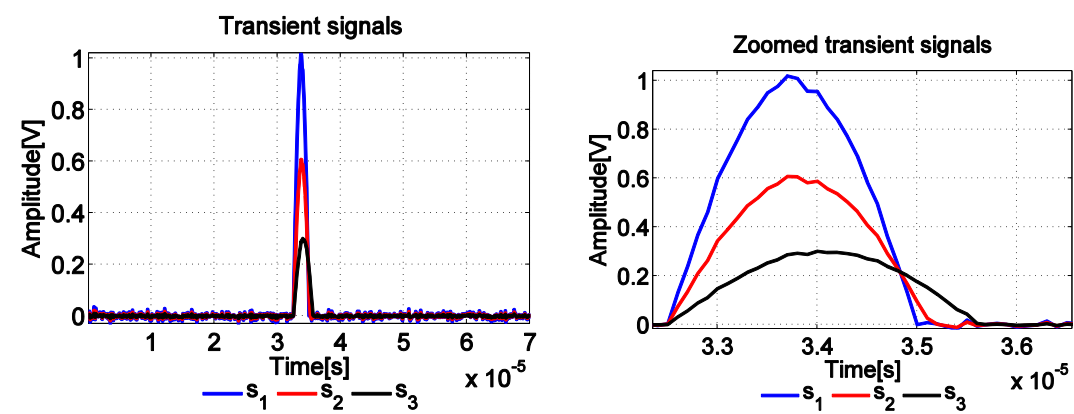

Fig. 2 Transient signals considered for the multi-lag phase-space analysis

The chosen signals from Fig. 2 seem to be quite similar, but, at a closer look, slight differences appear. Firstly, these signals are studied using the wavelet analysis [26, 27, 28].
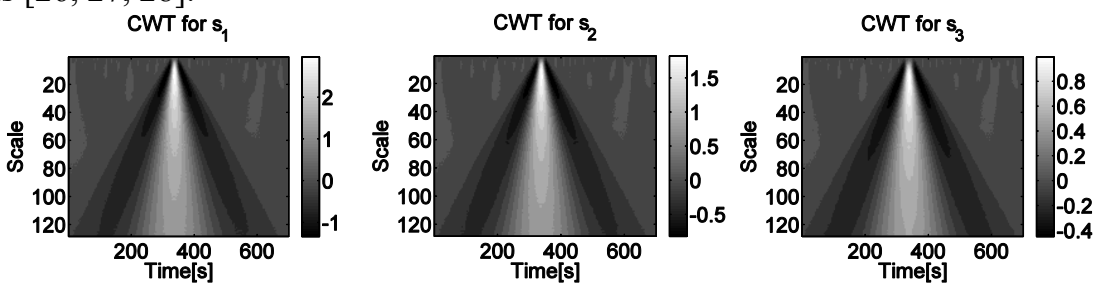

Fig. 3 The continuous wavelet transform (with the Mexican Hat mother-function ) applied on the three transient signals

From Fig. 3, it can be observed that the slight differences between the signals cannot be highlighted by the wavelet transform: their presence is detected, by their shape does not present any discriminating element.

In order to distinguish between these transient signals, the multi-lag phase space analysis is considered. The study of the representation of the trajectory for multiple lags is done by two approaches: the elliptic modeling of the trajectory for the area estimation in the phase-space, respectively, the polar coordinate representation.

The elliptic modeling supposes to determine the ellipse that circumscribes the phase-space trajectory and, therefore, to estimate the area of the trajectory through the ellipse's area computation. The area of the modeling ellipse is a new descriptor of the transient signal.

The trajectory is modeled by considering the solution that minimizes the following system [29]:

$$
S=\sum_{i=1}^{M}\left[F\left(x_{i}, y_{i}\right)\right]^{2}
$$


where $F(x, y)=\Gamma x^{2}+\Lambda y^{2}+1$. The least mean square estimation of eq.(12) gives the couple $(\Gamma, \Lambda)$. It goes that the major semi axes $a=1 / \Gamma$ and the minor semi axes $b=1 / \Lambda$.

The next step after the elliptic modeling is the area estimation and the estimation of the optimal delay. The delay is considered to be optimal for the value that provides an average value of the area and provides an adequate representation of the trajectory. This average value area assures a suitable phase-space representation where the trajectory does not evolve too close to the main diagonal (case of redundancy) or its evolution is too complicated (case of irrelevance) [30].
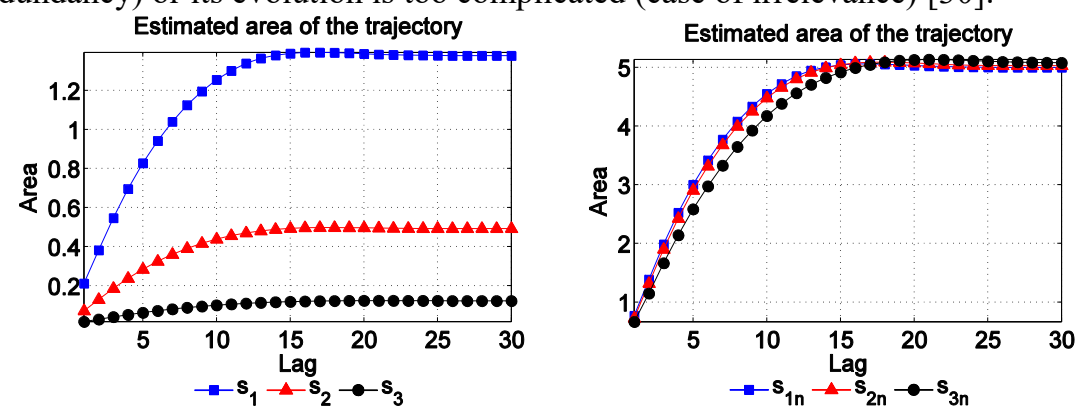

Fig. 4 The evolution of the area according to the lag (delay): for the signals presented in Fig. 2 (left figure) and for the normalized signals (right figure)

From Fig. 4, it can be noticed that the area of the signals has a similar trend with some differences for the normalized signals. Hereby, considering the average value of the area, $A=3$, the optimal delay for $s_{1}$ is $\tau_{1}=5$, for $s_{2}, \tau_{2}=5$ and for $s_{3}, \tau_{3}=6$.

With the chosen lags, the next step is to plot the phase-space trajectory into polar coordinates:

$$
\begin{aligned}
& \varphi[n]=\sqrt{x_{n}^{2}+y_{n}^{2}} \\
& \theta[n]=\arctan \frac{y_{n}}{x_{n}}
\end{aligned}
$$

This representation is very useful for signals with different amplitudes, because it shows the evolution of the position vector regardless of its length. 


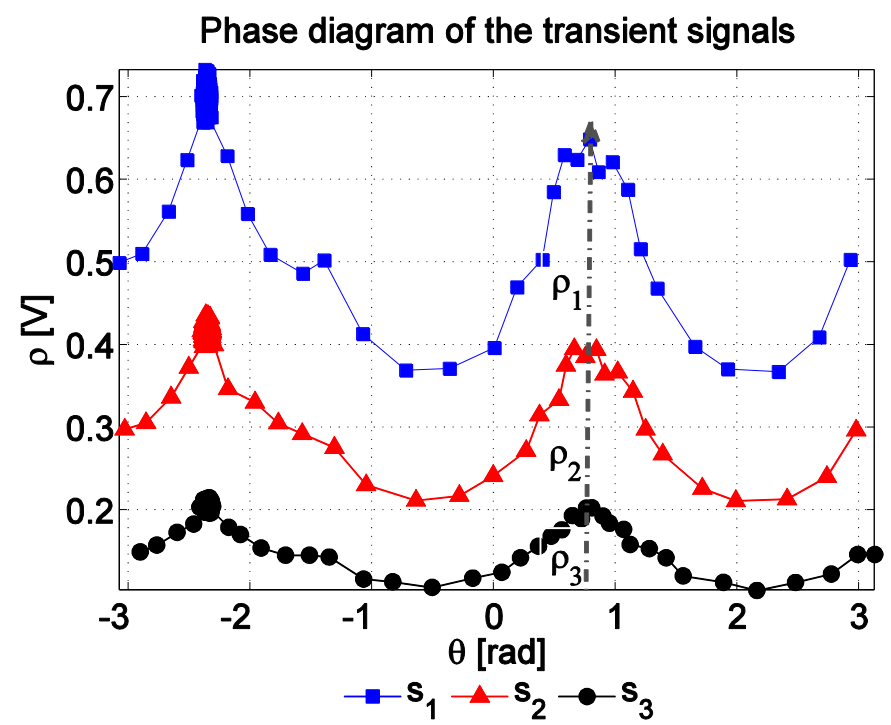

Fig. 5 The polar coordinates representation for the transient signals: $s_{1}: \tau_{1}=5, s_{2}: \tau_{2}=5$ and $s_{3}: \tau_{3}=6$

Fig. 5 shows the evolution of the transient signals on the phase diagram. It can be observed that the noise is concentrated in a small region of the phase space which is translated in a small points conglomeration around the peak corresponding to the angle $-3 \pi / 4$.

In this way, through the isolation of this region, the noise can be eliminated in the process of signal characterization. Moreover, the evolution of the trajectories is different for the peaks corresponding to the angle $\pi / 4$. Measuring the values of the maximum length of the vector corresponding to the $\pi / 4$ angle, it goes: $\rho_{1} / \rho_{2}=1.67$ and $\rho_{1} / \rho_{3}=3.43$. So, the ratio of the amplitude of the transient signals is then conserved in the phase diagram.

Furthermore, the number of points between the two angles $-3 \pi / 4$ and $\pi / 4$ (after excluding the points corresponding to the noise) is directly related to the fundamental frequency of the signal. Let $n_{0}$ be this number of points from the phase diagram. The fundamental period of the signal, $T_{0}$ is:

$$
T_{0}=\frac{2 n_{0}}{f_{s}}
$$

From eq. (14), it goes that: $f_{1} / f_{2}=1.04$ and $f_{1} / f_{3}=1.24$.

This results allow us to discriminate between the proposed signals although their characteristics are very close. 


\section{Characterization of partial discharges in high voltage cables}

In high voltage systems the presence of partial discharges (PD) are an indication of insulation weakness which, in time, may lead to total damage of the equipments [5]. Therefore, is it absolutely necessary to monitor such systems (power cables, transformers, etc.) in order to detect and localize the PD source, namely the position of the insulation troubles. Moreover, the characterization of these signals provides extra information regarding the long terms effects that they have upon the system [5].

The experiment was made on the grounding connection of the cable in order to record signals of $20 \mathrm{~ms}$ using high current inductive sensors and high speed data acquisition. It goes that each recorded signal has 2 million samples and a preliminary detection of the potential harmful zones is achieved using the spectrogram.

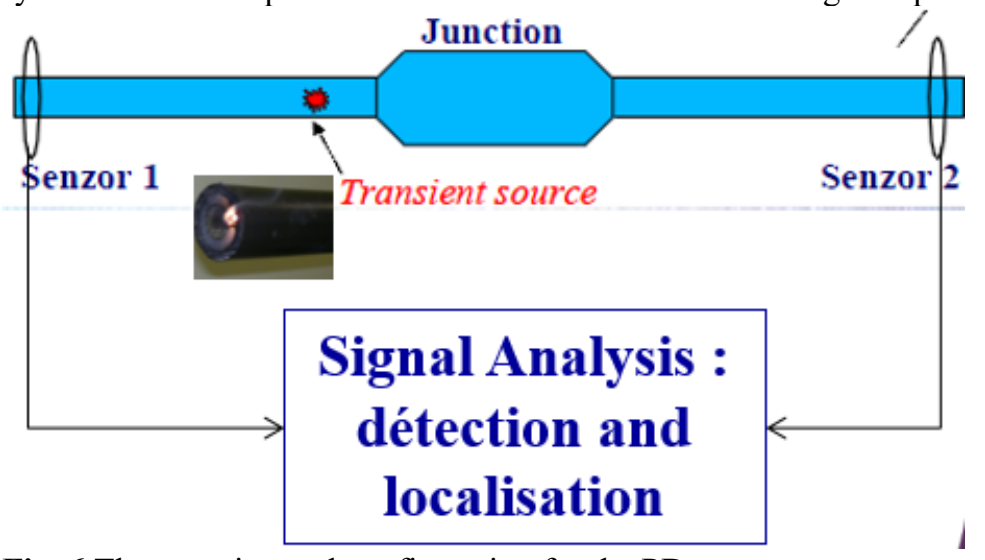

Fig. 6 The experimental configuration for the PD measurements

Then, the TDR measure is applied on partial discharges that have different SNRs

(a)

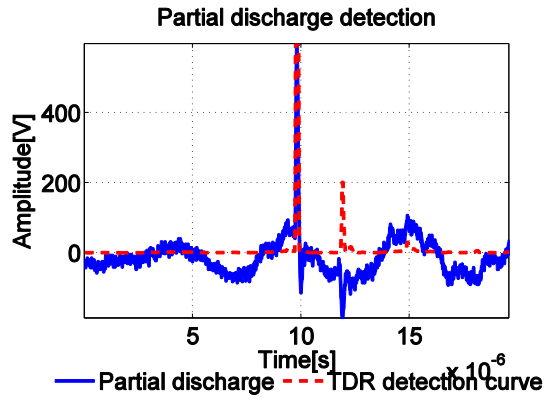

(b)

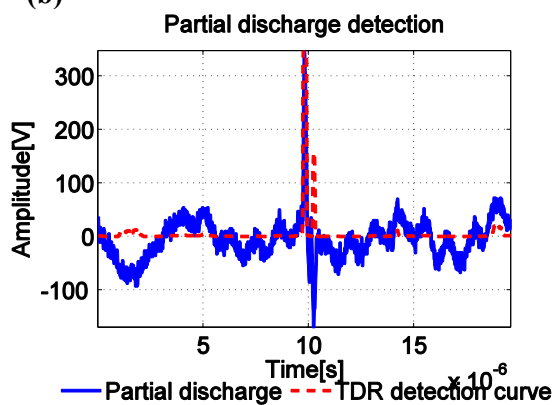


(c)

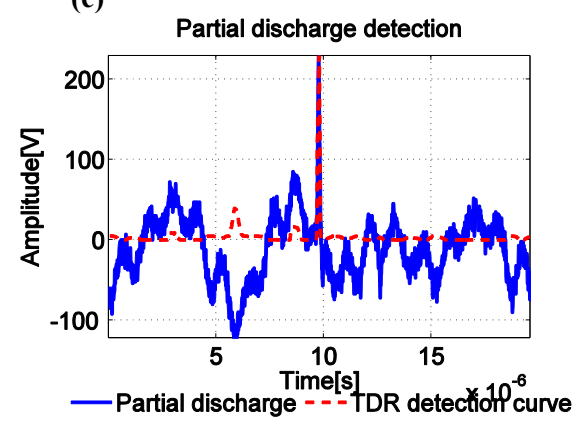

Fig. 7 Recorded partial discharges with different $S N R S$ and ratios $\alpha$ for $\varepsilon$ (eq. (7)): (a) $S N R=19.7 d B, m=3, d=3, \alpha=0.4$; (b) $\quad S N R=14 d B, m=3, d=4, \alpha=0.7$; (c) $S N R=8 d B, m=3, d=6, \alpha=0.95$

The PDs presented in Fig. 7 are detected with the TDR measure so that the noise has no impact upon the detection curve. Hereby, after the filtering of these signals, the filtered PDs have a $S N R$ improved with at least $20 \mathrm{~dB}$.

Next, these PDs are characterized using the multi-lag phase-space analysis. Firstly, the signals were normalized in order to eliminate the drawbacks that the different PDs amplitudes would involve. Then, on these signals, the area estimation is performed after the elliptic modeling. Fig. 8 presents the obtained results.

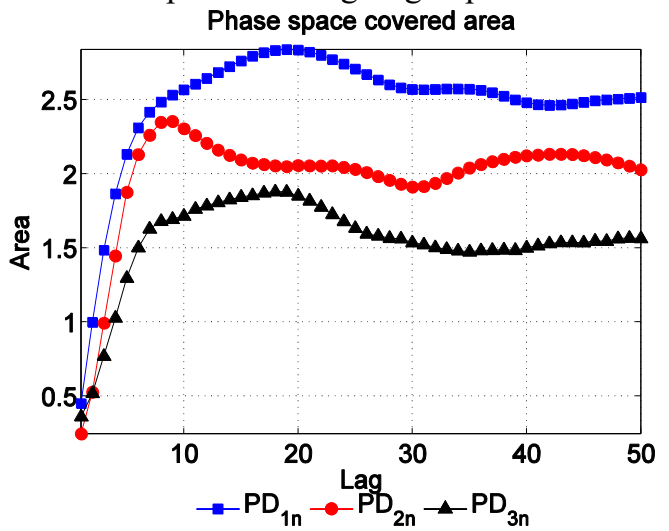

Fig. 8 The area estimation after the elliptic modeling in multi-lag representation

It can be observed that, even if the signals are normalized, their area evolution is different. The choice of the lag is done so that the area of the trajectory on the phase space has the same average value $(A=1.5): d_{P D_{1}}=3, d_{P D_{2}}=4$ and $d_{P D_{3}}=6$.

Using these lags, in Fig. 9 the phase diagram representation is presented. 


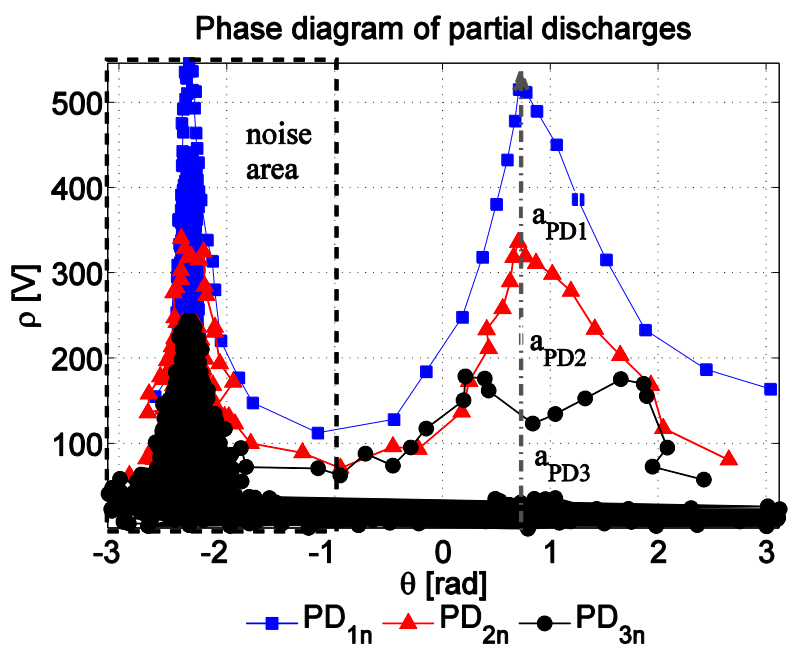

Fig. 9 The phase space representation in polar coordinates for: $P D_{1}: d_{P D_{1}}=3$, $P D_{2}: d_{P D_{2}}=4$ and $P D_{3}: d_{P D_{3}}=6$

The evolution of the trajectory is better pointed out in the $\pi / 4$ angle region than in the $-3 \pi / 4$ angle region. Moreover, the noise in distributed all along the peak corresponding to the $-3 \pi / 4$ angle depending on the $S N R$.

Recalling the fact that the amplitude ratio is conserved in the ratio between the lengths of the vectors corresponding to the $\pi / 4$ angle, means that: $a_{P D_{1}} / a_{P D_{2}}=1.53$ and $a_{P D_{1}} / a_{P D_{3}}=2.87$. Moreover, according to eq. (14), the frequency ratio are $f_{P D_{1}} / f_{P D_{2}}=1.15$ and $f_{P D_{1}} / f_{P D_{3}}=1.37$.

Hereby, the results show that the signals $P D_{2}$ and $P D_{3}$ suffer not only an attenuation, but also a frequency shift with respect to $P D_{1}$ which helps to establish their source characteristics.

\section{Electrical arcs in photovoltaic panels}

Photovoltaic panels are very important in the landscape of renewal energy sources of strategic interest, for both ecological reasons and the worldwide growing energy demand. The electrical arcs (EA) that appear in these systems can be a major problem, so it is necessary to supervise such phenomena in the system in order to keep it safe [7]. 
The experiment was performed with an electrical arc locator system composed of three acoustical microphones place in a $3 \mathrm{D}$ configuration and an wide band antenna placed in the center of the system (Fig. 10).
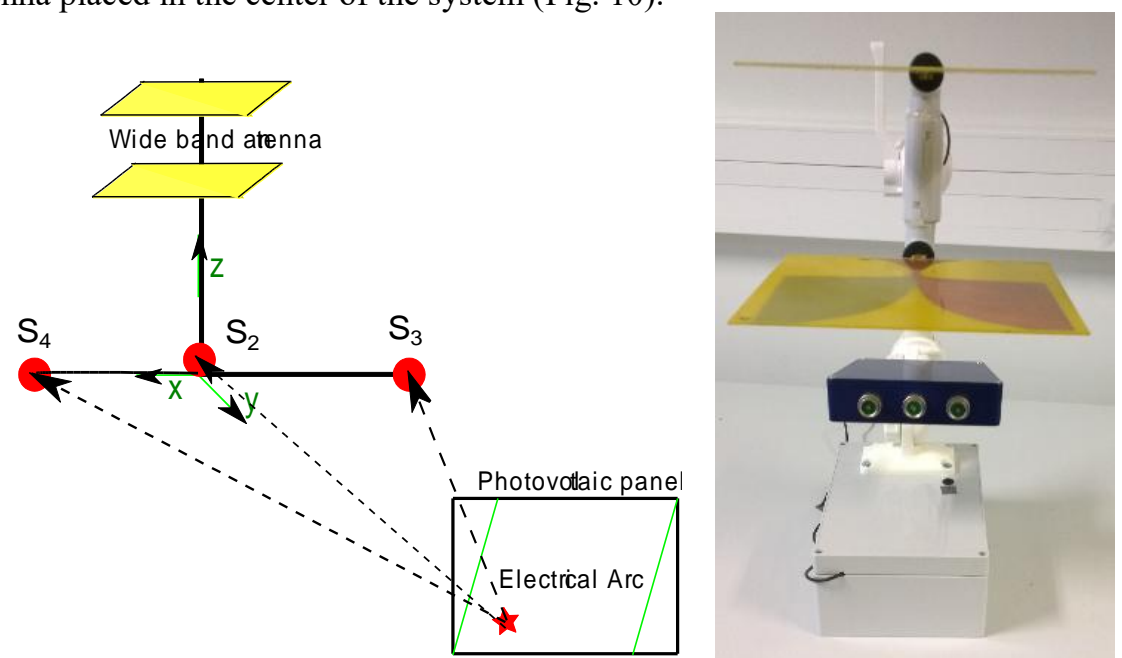

Fig. 10 The experimental configuration of the EA locator system

The detection and estimation of times of arrival of the transient signals generated by electrical arc at each sensor has been done using the TDR measure, the spectrogram and the wavelet transform. Fig. 11 presents the recorded signals and their detection curves obtained with the TDR measure.
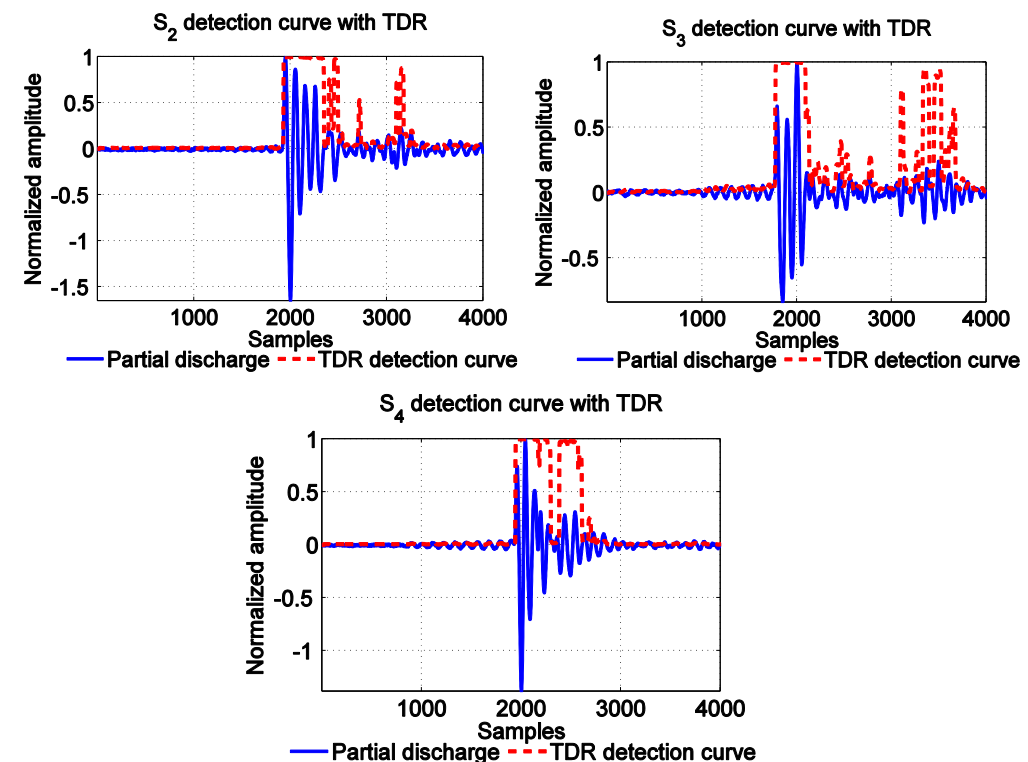
Fig. 11 The electrical arc locator system, the recorded AEs and the detection curve obtained with the TDR measure: $m=3 ; d=8 ; \alpha=0.7$

The recording is done at a sampling frequency of $f_{S}=5 \mathrm{MHz}$ for a period of $10 \mathrm{~ms}$ ( 50000 samples). In terms of location accuracy, the TDR measure is compared with the classical time-scale approaches. The spatial localization is achieved by solving the geometrical system (eq. (15)) based on the time-of-arrival (TOA) of the electrical arc at each microphone. The TOA is obtained by imposing the same threshold $(0.5)$ at the normalized detection curves based on each method.

$$
\left\{\begin{array}{l}
d_{P S_{2}}-d_{P S_{1}}=v \cdot t_{21} \\
d_{P S_{3}}-d_{P S_{1}}=v \cdot t_{31} \\
d_{P S_{4}}-d_{P S_{1}}=v \cdot t_{41}
\end{array}\right.
$$

where $d_{P S_{i}}=\sqrt{\left(x_{s_{i}}-x_{P}\right)^{2}+\left(y_{s_{i}}-y_{P}\right)^{2}+\left(z_{s_{i}}-z_{P}\right)^{2}}, i=\overline{1,4}$, each position of the acoustic microphone and antenna $\left(S_{1}\right)$ is known $S_{i}\left(x_{S_{i}}, y_{S_{i}}, z_{S_{i}}\right), t_{(i+1) i}$ is the time of arrival of the electrical arc at each microphone triggered by the $S_{1}$ wide band antenna.

The precision accuracy using each method is presented in Tab. 1.

\begin{tabular}{ll}
\hline Method & Relative error \\
\hline TDR measure & $6.2 \%$ \\
Wavelet & $11.2 \%$ \\
Spectrogram & $9.4 \%$ \\
\hline
\end{tabular}

Tab. 1 The spatial localization accuracy for the electrical arcs

In this application, the signal is propagated on multiple paths, because the experiment is performed in a closed facility: a $75 \mathrm{~m}^{2}$ laboratory sustained by 6 columns and equipped with test tables, desktops and test boards.

Although, the classical techniques detect and localize the electrical arc source in an effective manner, when it comes to multi-path signal discrimination, these methods are limited.

But, the multi-lag phase space analysis provides better results. Using one of the signals arrived at $S_{2}$ microphone, the reflections $s_{2}, s_{3}$ of the electrical arc are compared with the direct path signal $s_{1}$. 


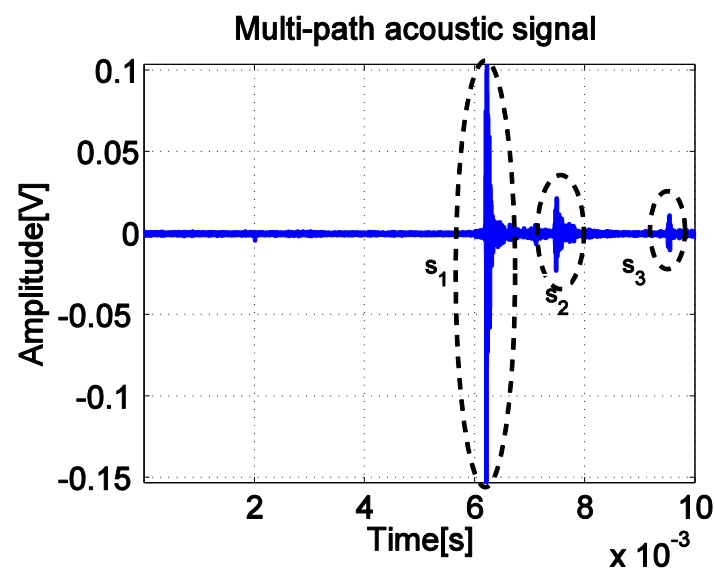

Fig. 12 The electrical arc recording $s_{1}$ and its reflections $s_{2}, s_{3}$

Therefore, for the signals highlighted in Fig. 12, the elliptic modeling is applied and the area of the estimated ellipse is determined. Previously, the signals are normalized in order to bring the signals at the same amplitude level.

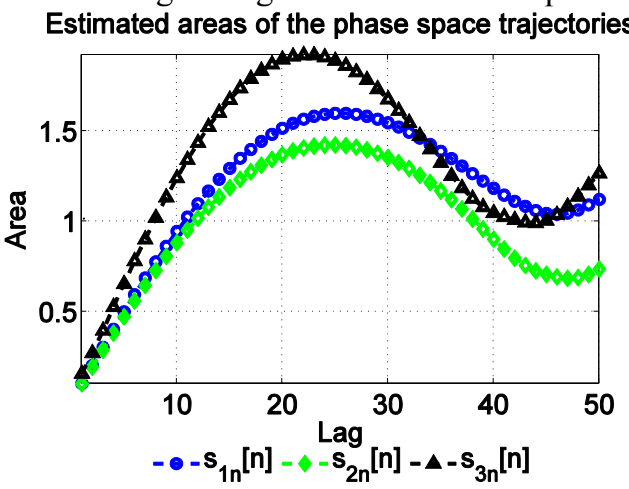

Fig. 13 The areas of the estimated ellipses that circumscribe the phase-space trajectory

The estimated ellipses evolve in a different manner, but they have the same trend. After an average value of the area is chosen, $A=1$, an optimal lag is determined: $d_{1}=11, d_{2}=12$ and $d_{3}=8$. The results of the phase diagram representation are shown in Fig. 14.
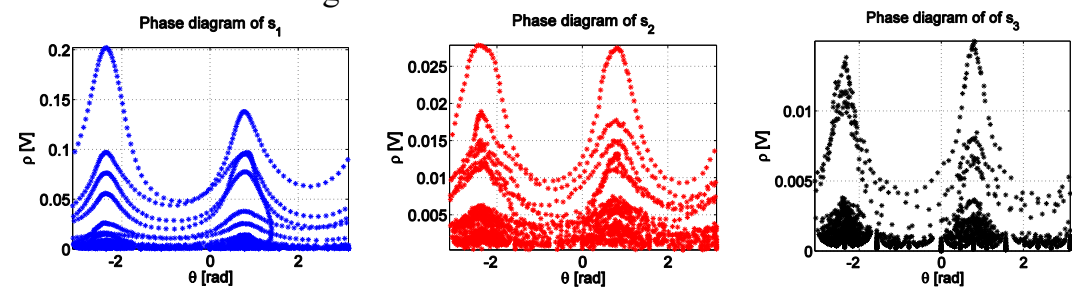
Fig. 14 The polar coordinates representation for the multi-path acoustic signals: $s_{1}: d_{1}=11, \quad s_{2}: d_{2}=12$ and $s_{3}: d_{3}=8$

In terms of attenuation, the phase diagram points out the same information as the time evolution of the signals. Moreover, the reflections present fewer curves that the direct path signal. This means that the reflections contain fewer oscillations than the direct signal, hereby, the reflections are dispersed.

Using eq. (14), it goes that: $f_{s_{1}} / f_{s_{2}}=0.94$ and $f_{s_{1}} / f_{s_{3}}=0.92$, meaning that the reflections suffer a time dilatation. Because the frequency ratios are close to 1 , it leads to the idea that the signals have the same source, but on their propagation path, the reflections are affected by multiple phenomena: diffusion, dispersion, attenuation, etc.

Concluding this part, the RPA approach provides a better localization accuracy than the time-scale methods and with the use of multi-lag phase-space analysis, it highlights new information regarding the characterization of transient signals and the changes that they suffer.

\section{Water hammer effect quantification}

The water hammer is a phenomenon that take place in a closed pipeline when a vane is suddenly closed while the water is flowing. The effect is that the liquid is forced to change its direction or to stop its motion. The risks of this operation vary from pipeline vibration to pipe collapse.

Usually, in industrial applications, this phenomenon is quantified using either intrusive pressure sensors, either a hydraulic formula (eq. (16)) with the condition that the characteristics of the system are well known [32, 33, 34].

$$
c=\frac{1}{\sqrt{\rho\left(\frac{1}{\varepsilon_{w}}+\frac{D}{e \cdot E}\right)}}
$$

where $c$ is the pressure wave speed, $\varepsilon_{w}$ is the bulk modulus of the fluid, $D$ is the pipe diameter, $e$ is the pipe wall thickness and $E$ is the Young modulus of the pipe.

The direct relation between the speed of the pressure wave and the pressure variation $\Delta p$ is given by Joukovski's equation [32]:

$$
\Delta p=\rho \cdot c \cdot v_{0}
$$

where $\rho$ is the fluid density and $v_{0}$ is the steady flow velocity.

Our approach consists in placing a pair of ultrasonic sensors on the pipe and to record the acoustical effect of the water hammer in order to compute the pressure 
wave speed. This approach has the advantage to supervise the system as it is and not to require any additional intrusive changes to the system as inserting a pressure sensor inside the pipe (eq. (17)) or determining the exact characteristics of the hydraulic system (eq. (16))

The experiment is done on a horizontal pipe supplied by a tank (ST) of $200 l$ volume. The pipe has a length of $L=10.11 \mathrm{~m}$, it is made from Plexiglas with an exterior section of $50 \times 50 \mathrm{~mm}^{2}$ and a circular interior section of $D=39 \mathrm{~mm}$. The water evacuation diaphragm has a diameter of $d=20 \mathrm{~mm}$.

Our ultrasonic transducers are placed on the pipe at a distance of $8 \mathrm{~cm}\left(\mathrm{~S}_{2}\right)$, respectively $16 \mathrm{~cm}$ ( $\left.S_{1}\right)$ from the closing vane $(\mathrm{CV})$. Next to them, the pressure sensor $\mathrm{P}$ is already installed in the pipe.

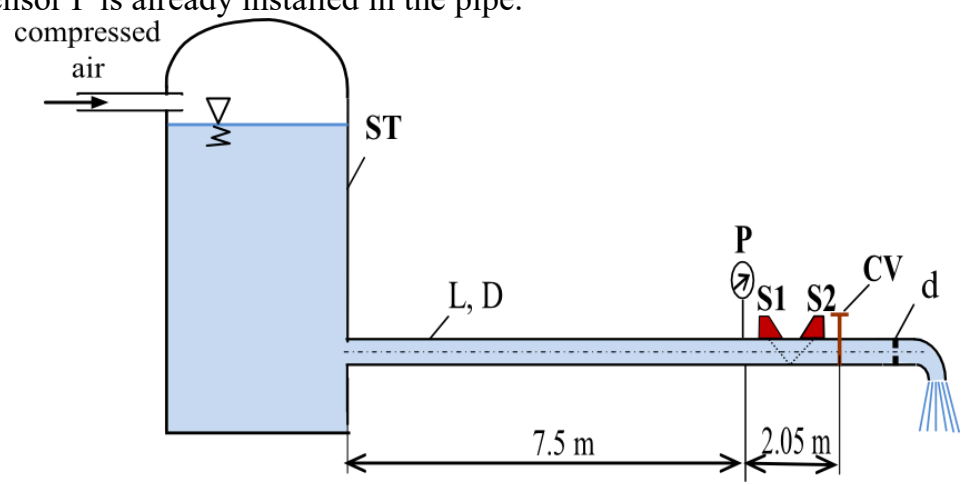

Fig. 15 The experimental configuration

The acquired signals, by both ultrasonic and pressure sensors are presented in Fig. 16. The highlighted areas emphasize that the acoustical effect happens simultaneously with the pressure variations.
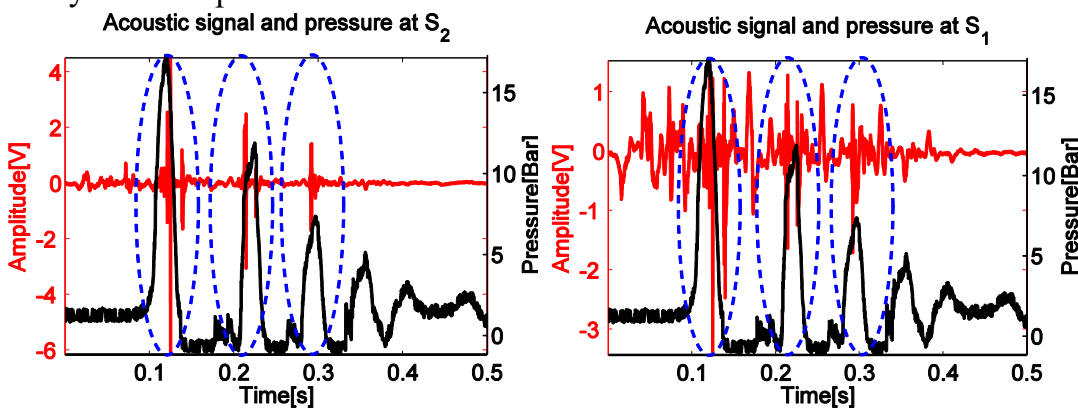

Fig. 16 The acoustic and pressure signals at sensor $S_{2}$ (left figure), respectively at sensor $S_{1}$ (right figure)

The acoustic signal arrived at sensor $S_{2}$ is more clearer than the one arrived at $S_{1}$, because the first one is closer than the second from the CV. This happens be- 
cause the wave starts to diffuse. The results obtained with the $T D R$ measure are shown in Fig. 17.
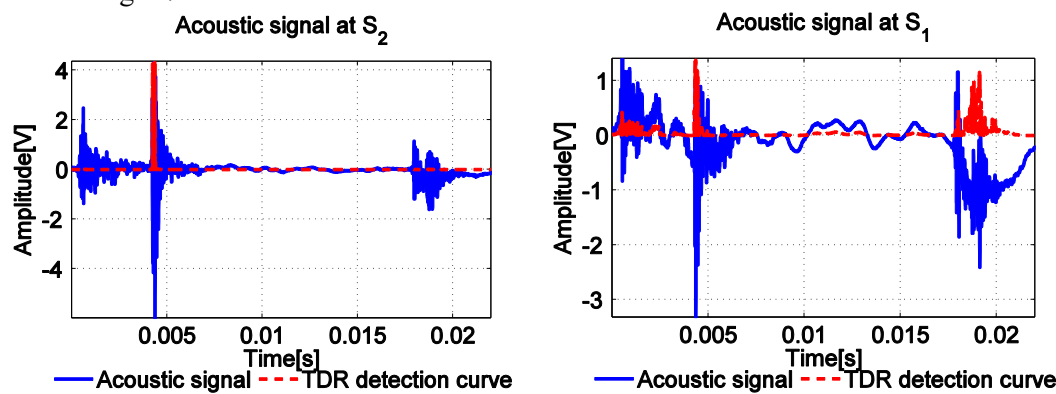

Fig. 17 The water hammer effect detection on the signals recorded by the two sensors: $m=3, d=10, \varepsilon=70$

Although, the effect of the mechanical vibrations of the pipe are also recorded by the sensors (especially sensor $S_{1}$ ), the transient detection is successfully obtained. After imposing a threshold for the $T D R$ measure of $80 \%$ from its maximum value, two times of arrival are determined. Hereby, the time difference of arrival obtained is $T D O A=82 \mu \mathrm{s}$. Therefore, the estimated wave speed is $c_{T D R}=975 \mathrm{~m} / \mathrm{s}$.

Computing only the $T D O A=99 \mu s$ for the maxima of the recorded signals, the estimated wave speed is $c_{p k}=805 \mathrm{~m} / \mathrm{s}$. The difference between these approaches is explained by the diffusion.

With the use of eq. (16) and considering $\rho=1000 \mathrm{~kg} / \mathrm{m}^{3}, \varepsilon_{w}=2.1 \mathrm{GPa}, e=10 \mathrm{~mm}$ and $E=5.66 \mathrm{GPa}$, the theoretical value of the wave speed is $c_{t h}=926 \mathrm{~m} / \mathrm{s}$.

Still, the pressure variation recorded by the pressure sensor $\mathrm{P}$ is $\Delta p=15.9 \mathrm{bar}$. Knowing that the flow rate of the water inside the pipe before the closure is $Q=1.98 \mathrm{l} / \mathrm{s}$, it goes that the speed of the water in steady state is $v_{0}=1.66 \mathrm{~m} / \mathrm{s}$. Making use of eq. (17), the experimental pressure wave speed is $c_{e}=959 \mathrm{~m} / \mathrm{s}$.

Relating the pressure wave speed the pressure variation using eq. (17), the relative error for the estimation of the pressure variation is performed, namely the water hammer effect is quantified.

\begin{tabular}{ll}
\hline Method & Relative error \\
\hline Experimental $\left(c_{e}\right)$ & - \\
TDR measure $\left(c_{T D R}\right)$ & $1.69 \%$ \\
\hline
\end{tabular}




\begin{tabular}{ll}
\hline Maximum peak $\left(c_{p k}\right)$ & $16.02 \%$ \\
Theoretical $\left(c_{t h}\right)$ & $3.43 \%$ \\
\hline
\end{tabular}

Tab. 2 The relative error using different approaches

The results from Tab. 2 state that the $T D R$ measure based on the RPA concept is very efficient in the water hammer effect quantification with an error below $2 \%$.

\section{Conclusions}

The concepts of RPA and phase space bring new insights which together with classical signal processing methods can help the analysis the transient signals.

The choice of the RPA concept has the advantage to be a data-driven method, therefore, it is an alternative to the classical transient signal processing techniques based on projection of analyzed signals on a given dictionary. Three applicative contexts have been addressed in our work.

The first one is the electrical partial discharge analysis. The detection method is based on the RPA method parameters using the TDR measure. Furthermore, the signals are characterized and discriminated using the concept of multi-lag phase space analysis. In addition, for the electrical arcs the detection and characterization is similarly obtained. The localization precision accuracy outperforms the classical non-stationary signals processing methods.

The third application, related to the water hammer phenomenon analysis, is pointed out through our ultrasonic non-intrusive approach that proves to be the closest to the reference method. The effect of the phenomenon is quantified using the $T D R$ measure which is more robust to the effect of pressure wave diffusion that takes place inside the pipe during the experiment.

Our future work foresees to correlate the information given by this new approach with the physical parameters of the system. For this purpose, our main research efforts will concentrate to the development of new descriptors of transients signals, derived from multi-lag phase diagram analysis. The characteristics of such descriptors that we look for are both the parsimony and the robustness to disturbing factors.

In parallel, new applications domains will be addressed aiming to provide new practically-oriented approaches for transient phenomena. 


\section{Acknowledgement}

This work has been supported in part by the Dema'Loc project funded by Institut Carnot "Energies du futur" and by the "Smart Hydro Monitoring" project (Rhone Alpes "Tenerrdis" and "Minalogic" research clusters).

\section{References}

[1] J. Gao, Y. Cao, W.W. Tung, J. Hu, "Multiscale Analysis of Complex Time Series: Integration of Chaos and Random Fractal Theory, and Beyond", John Wiley \& Sons Publishing, New Jersey, USA, 2007.

[2] A. Serbanescu, L. Cernaianu, C. Ivan, "New Approaches in Nonlinear Dynamics Analysis of Complex Systems and processes., International Symposium of Electronics, Computers and Artificial Intelligence, Pitesti, 2009.

[3] C. Ioana, A. Digulescu, A. Serbanescu, I. Candel, F.-M. Birleanu, "Recent Advances in Nonstationary Signal Processing Based on the Concept of Recurrence Plot Analysis", Springer Proc. in Mathematics \& Statistics, M. Marwan et al. (Eds.): "Translational Recurrences", pp. 75-93, Cham, Switzerland, 2014.

[4] I. Candel, A. Digulescu, A. Serbanescu, E. Sofron, " Partial discharge detection in high voltage cables using polyspectra and Recurrence Plot Analysis ", 9th International Conference on Communications (COMM), Bucharest, Romania, 2012.

[5] Boggs and G. C. Stone, "Fundamental limitations in the measurement of corona effect and partial discharges" Ontario Hydro Research, Toronto, Canada IEEE Transactions on Electrical Insulation Vol. EI-17 No.2, April 1982143.

[6] A. Digulescu, M. Paun, C. Vasile, T. Petrut, D. Deacu,C. Ioana, R. Tamas, "Electrical arc surveillance and localization system based on advanced signal processing techniques" IEEE International Energy Conference (ENERGYCON), Dubrovnik, Croatia, 2014.

[7] C. Strobl, P. Meckler, "Arc Faults in Photovoltaic Systems", Proc. 25th ICEC \& and 56th IEEE Holm Conference on Electrical Contacts, Charleston, 2010, pp. 216-222.

[8] F. M. Birleanu, I. Candel, C. Ioana, C. Gervaise, A. Serbanescu, G. Serban, ”A vector approach to transient signal processing", The 11th Conference on Information Science, Signal Processing and their Applications, Montreal, Canada, July 3-5 2012.

[9] N. Marwan, J. Kurths, "Nonlinear analysis of bivariate data with cross recurrence plots", Phys. Lett. A; 302:299-307, 2002.

[10] F.Popescu, F. Enache, I. C. Vizitiu, P. Ciotîrnae, "Recurrence Plot Analysis for Characterization of Appliance Load Signature", the 10th International Conference on Communications, Bucharest, Romania, 2014.

[11] H. Yang, "Multiscale recurrence quantification analysis of spatial cardiac vectorcardiogram (VCG) signals," IEEE Transactions on Biomedical Engineering, Vol. 58, No. 2, p339-347, 2011.

[12] Y. Chen, H. Yang, "Multiscale recurrence analysis of long-term nonlinear and nonstationary time series," Chaos, Solitons and Fractals, Vol. 45, No. 7, p978-987, 2012.

[13] G.M. Ramirez Avila, A. Gapelyuk, N. Marwan, H. Stepan, J. Kurths, T. Walther, N. Wessel, "Classifying healthy women and preeclamptic patients from cardiovascular data using recurrence and complex network methods", Autonomic Neuroscience: Basic and Clinical, 178 (1-2), pp. 103-110, 2013

[14] C. L. Webber Jr., J. P. Zbilut, "Recurrence quantification analysis of nonlinear dynamical systems", Tutorials in Contemporary Nonlinear Methods for the Behavioral Sciences Web 
Book, Eds.: M. A. Riley and G. C. Van Orden, National Science Foundation (U.S.), 26-94, 2005.

[15] J. P. Zbilut, C. L. Webber Jr., "Recurrence quantification analysis", Wiley Encyclopedia of Biomedical Engineering, Eds.: M. Akay, John Wiley \& Sons, 2006.

[16] J. Fan, Q. Yao, "Nonlinear Time Series: Nonparametric and Parametric Methods", Springer, New York, USA, 2005.

[17] J. C. Sprott, "Chaos and Time-Series Analysis", Oxford University Press, New York, USA, 2003.

[18] J. Gao, Y. Cao, L. Gu, J. Harris, J. Principe, "Detection of weak transitions in signal dynamics using recurrence time statistics", Phys. Lett. A 317, 64-72, 2003.

[19 ] J. Gao, Y. Cao, W.W. Tung, J. Hu, "Multiscale Analysis of Complex Time Series: Integration of Chaos and Random Fractal Theory, and Beyond", John Wiley \& Sons Publishing, New Jersey, USA, 2007.

[20] N. Marwan, S. Schinkel, J. Kurts, "Recurrence plots 25 years later - Gaining confidence in dynamic transitions," Europhysics Letters, 101, 20007, 2013

[21] N. Marwan, M. C. Romano, M. Thiel, J. Kurths, "Recurrence Plots for the Analysis of Complex Systems" Physics Reports, 438 (5-6), 237-329, 2007.

[22] H. Kantz, T. Schreiber, "Nonlinear Time Series Analysis", University Press, Cambridge, 1997.

[23] J. P. Eckmann, S. O. Kamphorst, D. Ruelle, "Recurrence Plots of Dynamical Systems", Europhysics Letters 5 (9): 973-977, 1987.

[24] Florin-Marian Birleanu, Cornel Ioana, Cedric Gervaise, Jocelyn Chanussot, Alexandru Serbanescu, and Gheorghe Serban, "On the recurrence plot analysis method behaviour under scaling transform," in 2011 IEEEWorkshop on Statistical Signal Processing (SSP2011), June 2011, pp. 789-792.

[25] S. Mallat, S. Zhong, "Characterization of signals from multiscale edges", IEEE Transaction on Pattern Analysis and Machine Intelligence, vol. 14, No. 7, p. 710-732, July 1992.

[26] S. Mallat, "A Wavelet Tour of Signal Processing, Second Edition”, Academic Press, 1999.

[27] S. Mallat, "A Theory for Multiresolution Signal Decomposition: The Wavelet Representation", IEEE Transactions on Pattern Analysis and Machine Intelligence, v.11 n.7, p.674-693, July 1989.

[28] M. Misiti, Y. Misiti, G. Oppenheim, J-M Poggi, "Wavelets Toolbox Users Guide”, the MathWorks, Wavelet Toolbox, for use with MATLAB, 2000.

[29] C. Bernard, T. Petrut, G. Vasile, C. Ioana, "Multi-Lag Phase Space Representations for Transient Signal Characterization”, Europeean Signal Processing Conference, 2014, Portugal, pp. $2115-2119$

[30] M. Casdagli, S. Eubank, J. Doyne Farmer, J. Gibson, "State space reconstruction in the presence of noise", Phys. D, 51, 1-3(1991), 52-98, 1991.

[31] I. Candel, F. Bunea, G. Dunca, D. M. Bucur, C. Ioana, B. Reeb, G. D. Ciocan, "Detection of cavitation vortex in hydraulic turbines using acoustic techniques", IOP Conference Series: Earth and Environmental Science, Volume 22, Erosion and Cavitating Flows.

[32] E. Benjamin Wylie, L. Victor Streeter, "Fluid Transients in Systems", Prentice-Hall, Inc., 1993.

[33] F. E. Hachem, A. J. Schleiss,"Effect of drop in pipe wall stiffness on water-hammer speed and attenuation", Journal of Hydraulic Research, Volume 50, Issue 2, 2012.

[34] N. H .C. Hwang, R. J. Houghtalen, "Fundamentals of Hydraulic Engineering Systems", Prentice Hall, Inc. 3ed.,1996.

[35] W.L. Rubin, "Radar-Acoustic Detection of Aircraft Wake Vortices", Journal of Atmospheric and Oceanic Technology, Vol. 17, pp. 1058-1065, 2000. 